\title{
Inhibition of proliferation, sprouting, tube formation and Tie2 signaling of lymphatic endothelial cells by the histone deacetylase inhibitor SAHA
}

\author{
HSUEH-TSEN CHENG ${ }^{1}$ and WEN-CHUN HUNG ${ }^{2}$ \\ ${ }^{1}$ Institute of Biomedical Sciences, National Sun Yat-Sen University, Kaohsiung 804; \\ ${ }^{2}$ National Institute of Cancer Research, National Health Research Institutes, Tainan 704, Taiwan, R.O.C.
}

Received January 17, 2013; Accepted March 27, 2013

DOI: $10.3892 /$ or.2013.2523

\begin{abstract}
Histone deacetylase (HDAC) inhibitors exert potent inhibitory effects on various types of human cancer. The pioneer drug suberoylanilide hydroxamic acid (SAHA) is currently used in the clinic for cancer treatment. However, the effect of SAHA on tumor lymphangiogenesis is unclear. We recently showed that SAHA suppresses the expression and production of pro-lymphagenic factor vascular endothelial growth factor-C (VEGF-C) in breast cancer cells. In the present study, the effect of SAHA on lymphatic endothelial cells (LECs) was examined. We generated a lymphatic-like endothelial cell line (named FP01) by overexpressing the master LEC transcription factor PROX1 in EA.hy926 endothelial cells. This cell line exhibited a gene expression pattern and phenotype similar to primarily cultured LECs. SAHA inhibited cell cycle progression and proliferation of FP01 cells. In addition, SAHA suppressed sprouting and tube formation in these cells. Moreover, SAHA attenuated the angiopoietin (Ang)/Tie signaling pathway which plays important roles in the regulation of LEC function. FP01 cells expressed Ang1, Ang2, Tie1 and Tie2, and SAHA dose-dependently reduced the expression of Tie 2 in these cells. Tie 2 promoter activity was attenuated by SAHA indicating a transcriptional repression. Importantly, Tie 2 protein was significantly reduced by SAHA at the concentration in which no alteration of Tie2 mRNA was detected. We found that SAHA enhanced Tie2 protein degradation via the ubiquitin-proteasome pathway, and the expression of c-Cbl, the E3 ligase for Tie2 ubiquitination, rapidly increased after SAHA treatment. Knockdown of c-Cbl reversed SAHA-induced Tie2 protein degradation.
\end{abstract}

Correspondence to: Professor Wen-Chun Hung, National Institute of Cancer Research, National Health Research Institutes, No. 367 Shengli Road, Tainan 704, Taiwan, R.O.C.

E-mail: hung1228@ms10.hinet.net

Key words: histone deacetylase, suberoylanilide hydroxamic acid, Tie2 tyrosine kinase, ubiquitin-proteasome pathway, c-Cbl
Taken together, our results demonstrate that SAHA impairs the proliferation, sprouting and tube formation of LECs and attenuates Ang/Tie signaling in LECs by downregulating Tie-2 via transcriptional and post-transcriptional mechanisms.

\section{Introduction}

Targeting of epigenetic alterations such as DNA methylation and histone modification is an important field for cancer research and for anticancer drug development $(1,2)$. One class of promising epigenetic drugs is histone deacetylase (HDAC) inhibitors. These inhibitors suppress the enzymatic activity of HDAC and induce re-activation of tumor-suppressor genes or downregulation of oncogenes to attenuate tumor growth. Among HDAC inhibitors, suberoylanilide hydroxamic acid (SAHA) is a pioneer drug that has been developed and is now approved for the treatment of cutaneous T cell lymphoma $(3,4)$. This drug is also undergoing clinical trials for the treatment of solid tumors. Several mechanisms are involved in the inhibition of tumor progression by SAHA. First, SAHA induces apoptosis in cancer cells by regulating pro-apoptotic or anti-apoptotic genes $(5,6)$. Second, SAHA inhibits cell cycle progression by increasing the expression of anti-proliferative genes such as cyclin-dependent kinase inhibitors (CDKIs) p21 and p27 to reduce cell growth $(7,8)$. Third, SAHA modulates the immune response to improve anticancer immune surveillance $(9,10)$. Fourth, SAHA suppresses proliferation of endothelial cells or induces their apoptosis to decrease tumor angiogenesis in vivo (11).

Recent studies demonstrate that induction of lymphangiogenesis is strongly associated with tumor metastasis and poor prognosis in cancer patients (12-14). Proliferation, maturation and remodeling of lymphatic vessels are controlled by different signaling pathways. Accumulating evidence suggests that angiopoietin (Ang)/Tie signaling is an important regulator in angiogenesis and lymphangiogenesis. The Ang family includes four ligands (Ang1, Ang2 and Ang3/4) and two cognate receptors (Tie1 and Tie2). Results of previous studies suggest that Ang1 mainly acts as a Tie 2 receptor agonist and Ang2 normally functions as an antagonist $(15,16)$. However, the role of Ang1 and Ang2 is cell context-dependent and Ang2 may function as a partial agonist under some physiological 
conditions $(17,18)$. Upon ligand stimulation, Tie2 forms a dimer or multimer and is autophosphorylated at tyrosine residues near its carboxyl terminus (19). Activated Tie2 then transmits the signaling via several downstream molecules including downstream of tyrosine kinase-related protein (DOKR), endothelial nitric oxide synthase (eNOS), SH2 domain-containing phosphatase (SHP2), growth factor receptor-bound protein 2 (GRB2) and the p85 subunit of PI3K to elicit different biological processes $(20,21)$. Mice lacking Ang2 exhibit major lymphatic vessel defects suggesting the importance of Ang2 in lymphatic function (22). Interestingly, Ang1 has also been demonstrated to promote lymphatic vessel formation and can rescue the lymphatic defect of Ang2-mutant mice (23). In addition, Ang1/Tie2 signaling enhances lymphatic integrity by modulating tight junction molecule expression during inflammation (24). Although SAHA has been shown to inhibit tumor angiogenesis, its effect on tumor lymphangiogenesis and the Ang/Tie system is still unclear. In this study, we addressed this issue and tried to elucidate the underlying mechanism.

\section{Materials and methods}

Generation of the Proxl-expressing lymphatic-like endothelial cell line and cell culture. Human endothelial EA.hy926 cells were a gift from Dr Ming-Hong Tai (National Sun Yat-Sen University, Taiwan). These cells were grown in DMEM/high glucose (Invitrogen Life Technologies, Carlsbad, CA, USA) medium containing $10 \%$ FBS, antibiotics and $25 \mathrm{mM}$ HEPES. EA.hy926 cells were transfected with the pcDNA-Prox1 expression vector (provided by Dr You-Hua Xie, Shanghai Institute of Biological Sciences, Shanghai, China). After $48 \mathrm{~h}$, cells were selected with G418 for 3 weeks, and a stable cell line FP01 was generated as previously described (25). Human primary lymphatic endothelial cells (LECs) were purchased from PromoCell (Heidelberg, Germany). LECs were grown in endothelial cell growth medium (MVII) with supplement mixture (PromoCell). All cell lines were cultured at $37^{\circ} \mathrm{C}$ in a $\mathrm{CO}_{2}$ incubator.

Reagents and antibodies. SAHA was purchased from LC Laboratories (Woburn, MA, USA). MG-132, chloroquine, mithramycin A, leupeptin and the anti-actin antibody were purchased from Sigma-Aldrich (St. Louis, MO, USA). Antibodies against Tie2 and Sp1 were obtained from Millipore (Billerica, MA, USA). Antibody against c-Cbl was purchased from Gene Tex, Inc. (San Antonio, TX, USA). Antibodies against cyclin E, cdc2, cdk2, p21 and p27 were purchased from Santa Cruz Biotechnology, Inc. (Santa Cruz, CA, USA). Antibodies against cyclin D1, cyclin A and cyclin B were obtained from Cell Signaling Technology (Beverly, MA, USA). Vascular endothelial growth factor (VEGF)-C (Cys156Ser) was purchased from R\&D Systems (Minneapolis, MN, USA).

RNA interference. c-Cbl and control luciferase shRNA interference vectors were obtained from National RNAi Core Facility (Institute of Molecular Biology, Academia Sinica, Taipei, Taiwan) and the targeting sequences were: $\mathrm{pLKO} .1-\mathrm{shCbl}$ (target sequence 5'-CCAGTGAGTTGGGAG TTATTA-3') and pLKO.1-shLuc (target sequence 5'-CTTCGAAATGTCCGT TCGGTT-3').
Transient cell transfection. Cells were seeded in 6-well plates. After overnight incubation, the GFP-Sp1 expression vector (provided by Dr Jan-Jong Hung, National Cheng Kung University, Taiwan) and the control vector were transfected into cells using Lipofectamine ${ }^{\circledR} 2000$ (Invitrogen Life Technologies). After $48 \mathrm{~h}$, cells were treated with SAHA for an additional $24 \mathrm{~h}$ and then harvested for different analyses.

Construction of Tie-2 promoter and luciferase assay. The genomic DNA was isolated from EA.hy926 cells by using the genomic DNA extraction kit (Qiagen, Hilden, Germany), and the $-703 /+232$ region from transcription start site of Tie 2 gene (NM_000459) was amplified by PCR using two specific primers: Tie2-703 forward, 5'-ATACTCGAGCTTGGGGCTA CATTGAGCAT-3' and reverse, 5'-ATTAAGCTTCACAGA GCCTTTGCATTTCA-3'. The amplified DNA fragment was subcloned into the luciferase reporter gene vector pGL3 (Promega, Madison, WI, USA) by XhoI and HindIII to yield the luciferase reporter construct pGL3-Tie2-(-703/+232). Using this construct as a template, two 5'-deletion constructs were generated by the following primers: Tie2-357 forward, 5'-AAA CTCGAGTACAGCAGCAGCAAAAGCAG-3' and Tie2-138 forward, 5'-ATACTCGAGGTTCCTTCTTGCCTCTAACTT GT-3'. FP01 cells were seeded into 6-well plates and transfected with $1 \mu \mathrm{g}$ of serial Tie-2 promoter-luciferase plasmids. After $24 \mathrm{~h}$, cells were incubated with various concentrations of SAHA for an additional $24 \mathrm{~h}$. The luciferase activity was detected by a reporter assay system according to the manufacturer's instructions (Promega), and the results were normalized to the protein concentration in cell lysates.

Reverse transcription-polymerase chain reaction (RT-PCR). Cells were treated with different concentrations of SAHA for $24 \mathrm{~h}$. Total RNA was isolated by using the RNeasy Mini kit (Qiagen), and mRNAs were reverse-transcribed to cDNA by M-MLV reverse transcriptase (Promega), using oligo-dT primers according to the manufacturer's instructions. The conditions of the PCR reaction included an initialization step for $5 \mathrm{~min}$ at $95^{\circ} \mathrm{C}, 30$ cycles of amplification $\left(1 \mathrm{~min}\right.$ at $95^{\circ} \mathrm{C}$ for denaturation, $1 \mathrm{~min}$ at $60^{\circ} \mathrm{C}$ for annealing and $1 \mathrm{~min}$ at $72^{\circ} \mathrm{C}$ for elongation) and $7 \mathrm{~min}$ at $72^{\circ} \mathrm{C}$ for final extension. The PCR primers were: Tie2 forward, 5'-AGTTCGAGGAGAGGC AATCA-3' and reverse, 5'-CCGAGGTGAAGAGGTTTCCT-3'; Tie1 forward, 5'-TTTAACCCTGGTGTGCATCC-3' and reverse, 5'-CCGCAGAAAATCTAGCAGGT-3'; Ang1 forward, 5'-TATGCCAGAACCCAAAAAGG-3' and reverse, 5'-GGGCACATTTGCACATACAG-3'; Ang2 forward, 5'-TGG GATTTGGTAACCCTTCA-3' and reverse, 5'-CCTTGAGCG AATAGCCTGAG-3'; c-Cbl forward, 5'-CCATGGCTCTGA AATCCACT-3' and reverse 5'-GGAGAATGTTCCCATCAG CA-3'; GAPDH forward, 5'-TGGGGAAGGTGAAGGTCGG AGTC-3' and reverse 5'-TCCCGTTCTCAGCCTTGACGG-3'.

Protein stability assay. Cells were cultured in the media containing various concentrations of SAHA for $24 \mathrm{~h}$ and then treated with $10 \mu \mathrm{g} / \mathrm{ml}$ of cycloheximide to block protein synthesis. Cellular proteins were harvested at different times after cycloheximide addition, and the Tie2 protein level was detected by western blotting. Tie 2 protein level in the cells collected at time zero was defined as $100 \%$. 


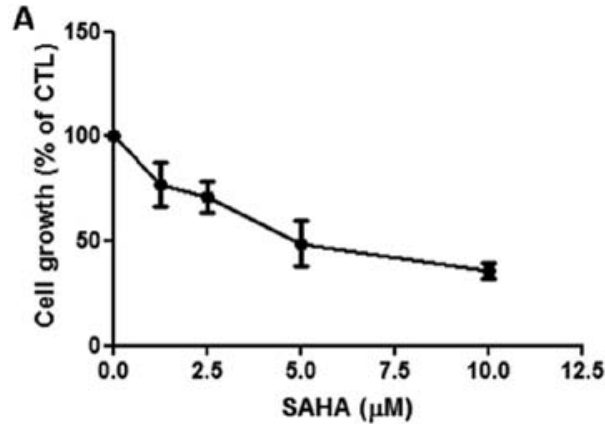

B

\begin{tabular}{|l|l|l|l|}
\hline & G0/G1 & S & G2/M \\
\hline SAHA $0 \mu \mathrm{M}$ & $67.7 \pm 1.9$ & $18.1 \pm 0.7$ & $14.6 \pm 1.1$ \\
\hline SAHA $125 \mu \mathrm{M}$ & $72.1 \pm 3.9$ & $12.7 \pm 3.9$ & $16.0 \pm 1.7$ \\
\hline SAHA $2.5 \mu \mathrm{M}$ & $722 \pm 3.0$ & $9.4 \pm 1.9$ & $18.7 \pm 3.4$ \\
\hline
\end{tabular}

C

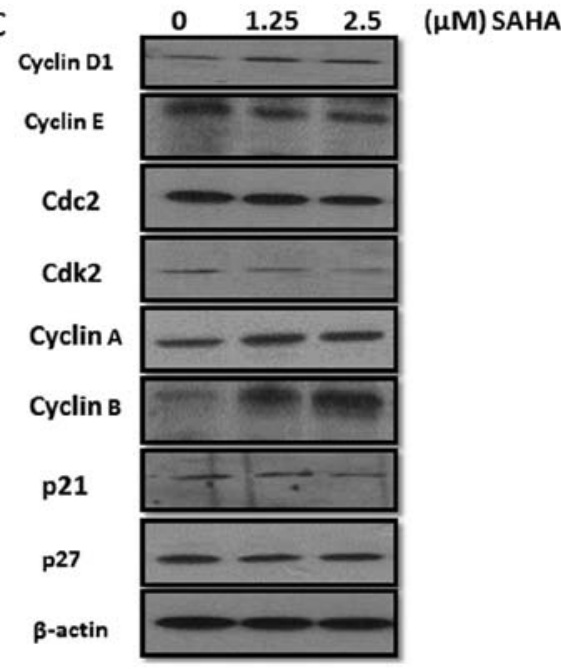

Figure 1. SAHA-inhibited cell cycle progression in FP01 cells is associated with upregulation of cyclins. (A) FP01 cells were treated with different concentrations of SAHA for $48 \mathrm{~h}$, and an MTT assay was performed to study cell growth. (B) Cells were treated with $0,1.25$ or $2.5 \mu \mathrm{M}$ of SAHA for $48 \mathrm{~h}$ and harvested for flow cytometric analysis. Results from three independent experiments are expressed as the mean \pm SE. (C) Cellular proteins were also harvested for SDS-PAGE. The protein level of cell cycle regulators was detected by western blotting.

Western blotting. Cells were treated with different concentrations of SAHA for $24 \mathrm{~h}$. After treatment, cells were washed with cold PBS and lysed by RIPA buffer (50 $\mathrm{mM}$ Tris- $\mathrm{HCl}$, $\mathrm{pH} 7.4,50 \mathrm{mM} \mathrm{NaCl}, 1 \mathrm{mM}$ EDTA, $0.5 \mathrm{M}$ sucrose, $0.25 \%$ sodium deoxycholate, $10 \%$ glycerol, $1 \%$ NP-40 and protease inhibitors) on ice for $10 \mathrm{~min}$. Cell debris was removed by centrifugation at $13,000 \mathrm{rpm}$ at $4^{\circ} \mathrm{C}$ for $10 \mathrm{~min}$. Cell lysates were subjected to SDS-PAGE separation and subsequently transferred onto a polyvinylidene difluoride membrane (Millipore). The membranes were incubated with 5\% non-fat milk in Tris-buffered saline and were probed with various primary antibodies. After extensive washing, the membranes were incubated with horseradish peroxidase-conjugated secondary antibodies and developed by enhanced chemiluminescence (ECL; Millipore) reagent according to the manufacturer's instructions.

Cell proliferation and flow cytometry. For cell proliferation assay, cells were seeded at a density of 7,500 cells/well in 96-well plates. After overnight incubation, cells were treated with different concentrations of SAHA for $48 \mathrm{~h}$. MTT assay was carried out according to the manufacturer's instructions to investigate cellular growth. For flow cytometric analysis, cells were cultured in the medium containing various concentrations of SAHA for $48 \mathrm{~h}$, washed with cold PBS and fixed in $70 \%$ ethanol overnight. Subsequently, cells were washed with cold PBS and stained with PI solution containing $20 \mu \mathrm{g} / \mathrm{ml}$ propidium iodide, $200 \mu \mathrm{g} / \mathrm{ml}$ RNase A and $0.1 \%$ Triton X-100 for $30 \mathrm{~min}$. Cell cycle analysis was performed by using Epics XL-MCL flow cytometry (Beckman Coulter, Miami, FL, USA).

Tube formation and sprouting assay. Tube formation and sprouting assays were performed as previously described (25). For the sprouting assay, 10,000 cells suspended in medium containing VEGF-C (Cys156Ser) and different concentrations of SAHA were mixed with Matrigel and added into 96-well plates. After $24 \mathrm{~h}$, cells sprouting in the three dimensional culture of Matrigel were observed by a microscope. One hundred cells were counted, and the percentage of cells with sprouting was expressed as the means \pm SE. For the tube formation assay, 20,000 cells suspended in medium containing VEGF-C (Cys156Ser) and different concentrations of SAHA were added into 96-well plates precoated with Matrigel. The formation of the tube-like structures was detected at 8 and $24 \mathrm{~h}$ after cell seeding. The number of vessel joints in 5 light microscopic fields was counted by using the Angiogenesis Image Analyzer V.2.0.0 software (Kurabo Industries, Osaka, Japan). Results from three independent experiments are expressed as the mean \pm SE.

Statistical analysis. All data are expressed as the means \pm SE. The Student's t-test was used to evaluate the differences between various experimental groups. P-value $<0.05$ was considered to indicate a statistically significant result.

\section{Results}

SAHA inhibits the proliferation of LEC-like FP01 cells. Culture of primary LECs is difficult as only a limited cell number can be isolated by using specific markers such as LYVE-1. To study LEC function, we recently established an LEC-like cell line (FP01) by overexpressing the master LEC transcription factor PROX1 in EA.hy926 endothelial cells (25). FP01 cells exhibited a gene expression pattern similar to primary LECs. In addition, these cells expressed vascular endothelial growth factor receptor 3 (VEGFR3) and their proliferation, sprouting and tube formation were strongly stimulated by VEGF-C (25). Our results suggest that the FP01 cell line is a useful model for functional investigation of LECs. As shown in Fig. 1A, SAHA dose-dependently inhibited the proliferation of FP01 cells and this drug at $10 \mu \mathrm{M}$ suppressed cell growth by $60-70 \%$. Flow cytometric analysis demonstrated that SAHA increased the percentage of cells at the G0/G1 and G2/M phases (Fig. 1B). Conversely, the percentage of cells at $\mathrm{S}$ phase was significantly reduced. Expression of CDKIs (p21 and p27) was not 
A

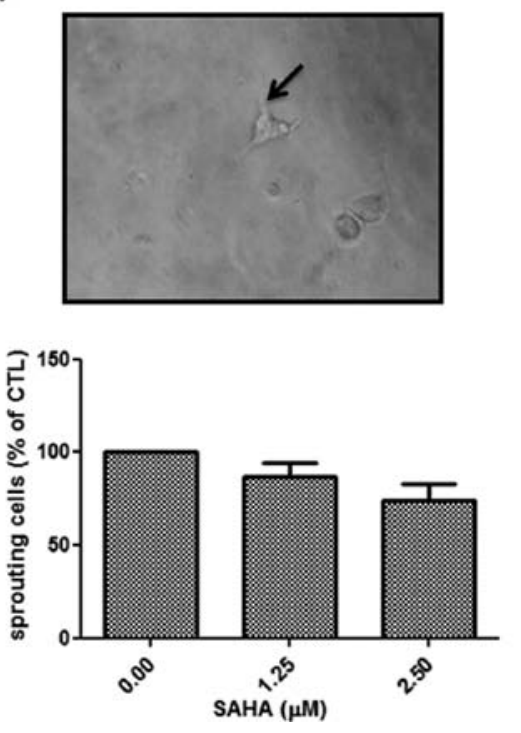

B
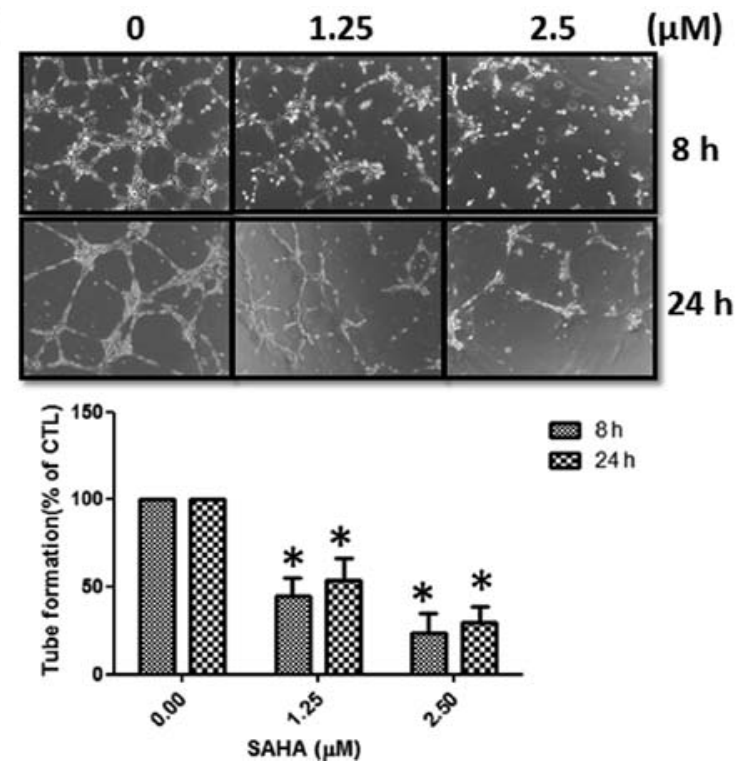

Figure 2. Inhibition of sprouting and tube formation of FP01 cells by SAHA. (A) Cells were cultured in 3D Matrigel containing VEGF-C (Cys156Ser) and 0 , 1.25 or $2.5 \mu \mathrm{M}$ SAHA. One hundred cells were counted, and the percentage of cells with sprouting (arrow) was expressed as the mean $\pm \mathrm{SE}$. "P $<0.01$ when compared to the control group. (B) A total of 20,000 cells was suspended in medium containing VEGF-C (Cys156Ser), and different concentrations of SAHA were added into 96-well plates precoated with Matrigel. The formation of tube-like structure was detected at 8 and $24 \mathrm{~h}$ after cell seeding. The number of vessel joints in 5 light microscopic fields was counted by using the Angiogenesis Image Analyzer V.2.0.0 software. The results from three independent experiments are expressed as the mean $\pm \mathrm{SE}$. ${ }^{*} \mathrm{P}<0.01$ when compared to the control group.

changed by SAHA (Fig. 1C). Interestingly, cyclin D1 and B1 were increased while cyclin $\mathrm{E}$ was reduced. Since constitutive expression of cyclin D1 and B1 will prevent G1/S progression and mitotic exit, our data suggest that SAHA-induced G0/G1 and $\mathrm{G} 2 / \mathrm{M}$ cell accumulation may be associated with upregulation of cyclin D1 and B1. In addition, reduction in cyclin E reflected the decrease of cells at $\mathrm{S}$ phase.

SAHA inhibits sprouting and tube formation of FP01 cells. For induction of lymphangiogenesis, LECs need to spout from existing lymphatic vessels and then organize into a new tube network structure. Treatment of SAHA repressed the sprouting of FP01 cells in the 3D culture (Fig. 2A). In addition, organization of the tube network structure of FP01 cells on matrix-coated plates was also significantly inhibited (Fig. 2B). Our data demonstrated that SAHA at the concentration of $2.5 \mu \mathrm{M}$ inhibited the number of vessel joints by $70-80 \%$ (Fig. 2B).

SAHA inhibits Tie2 transcription. The Ang/Tie signaling pathway is important for LEC maturation and biological function. As shown in Fig. 3A, FP01 cells expressed Ang1, Ang2, Tie1 and Tie2. Interestingly, we found that SAHA repressed Tie2 expression in a dose-dependent manner while Tie1 and Ang2 were not affected and Ang1 was only marginally reduced. We also used primary cultured LECs to confirm our results. Consistent with the data of FP01 cells, only Tie2 expression was significantly inhibited by SAHA (Fig. 3B). Western blot analysis demonstrated that the Tie2 protein level was dramatically reduced by SAHA in FP01 cells (Fig. 3C). Since Tie2 mRNA was attenuated, a direct inhibition of Tie2 transcription by SAHA was investigated. We cloned the proximal promoter region of the human Tie2 gene and generated a series of deletion mutants of the promoter-luciferase reporter (Fig. 4A). The reporter containing the $-703 /+232$ region of the human Tie2 gene exhibited high luciferase activity in FP01 cells indicating that this region is the major regulatory region of Tie2 expression in endothelial cells (Fig. 4B). These data were consistent with our RT-PCR data showing a high level of Tie2 mRNA in FP01 cells. Deletion mutant containing the $-357 /+232$ region was also repressed by SAHA. Conversely, luciferase activity of the deletion mutant containing the $-138 /+232$ region was very low and was not affected by SAHA suggesting that the $-357 /-138$ promoter region is important for Tie2 transcription in FP01 cells and is responsible for the inhibition by SAHA (Fig. 4D). Bioinformatics prediction revealed several transcription factor binding sites including $\mathrm{Sp} 1, \mathrm{Sp} 3$, $\mathrm{NF}-\mathrm{\kappa B}$ and Ets-1 in this region. However, ectopic expression of Sp1 or Ets-1 could not rescue downregulation of Tie2 by SAHA (Fig. 4C). These data indicate that SAHA inhibits Tie2 transcription via the -357/-138 promoter region and this effect is independent of $\mathrm{Sp} 1$ and Ets-1.

Induction of Tie 2 protein degradation via the ubiquitin/ proteasome pathway by SAHA. Although SAHA directly inhibits Tie2 gene transcription, our data demonstrated that the reduction in Tie2 protein was more significant than that of Tie 2 mRNA. In addition, SAHA at the concentration of $1.25 \mu \mathrm{M}$ did not affect the Tie2 mRNA level while it dramatically reduced Tie 2 protein level (Fig. 3A and C). These results suggest a transcription-independent inhibitory effect of SAHA on Tie 2 expression. We blocked protein synthesis by cycloheximide and examined Tie2 protein stability in control and SAHA-treated cells. Our results showed that SAHA reduced Tie2 protein by $40 \%$ at $13 \mathrm{~h}$ after exposure to cycloheximide (Fig. 5A and B). Conversely, no significant reduction in Tie2 
A FP01 cells

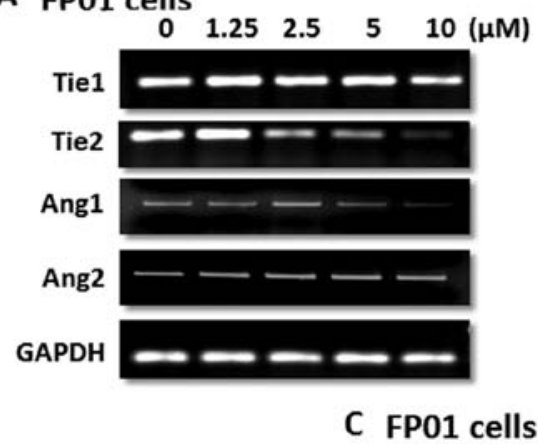

B Primary LECs

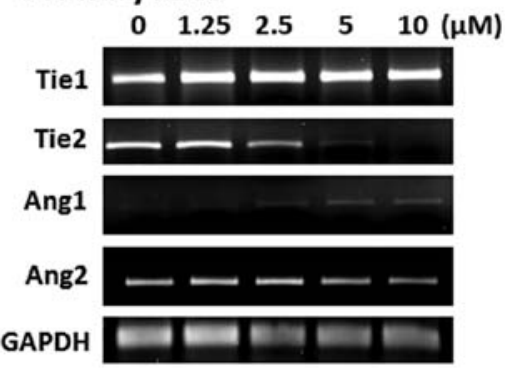

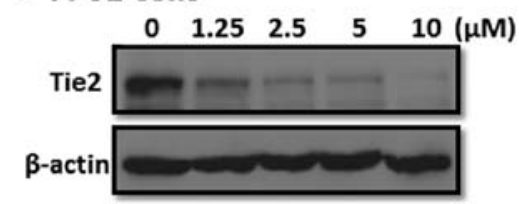

Figure 3. Inhibition of Tie2 expression in FP01 cells and primary LECs. (A) FP01 cells were treated with different concentrations of SAHA for 24 h and total RNA was isolated. Expression of Ang/Tie family genes was studied by RT-PCR. (B) Primary LECs were treated with different concentrations of SAHA for $24 \mathrm{~h}$, and the mRNA level of Ang/Tie family genes was studied by RT-PCR. (C) The Tie2 protein level of FP01 cells treated with different concentrations of SAHA was investigated by western blot analysis.

A
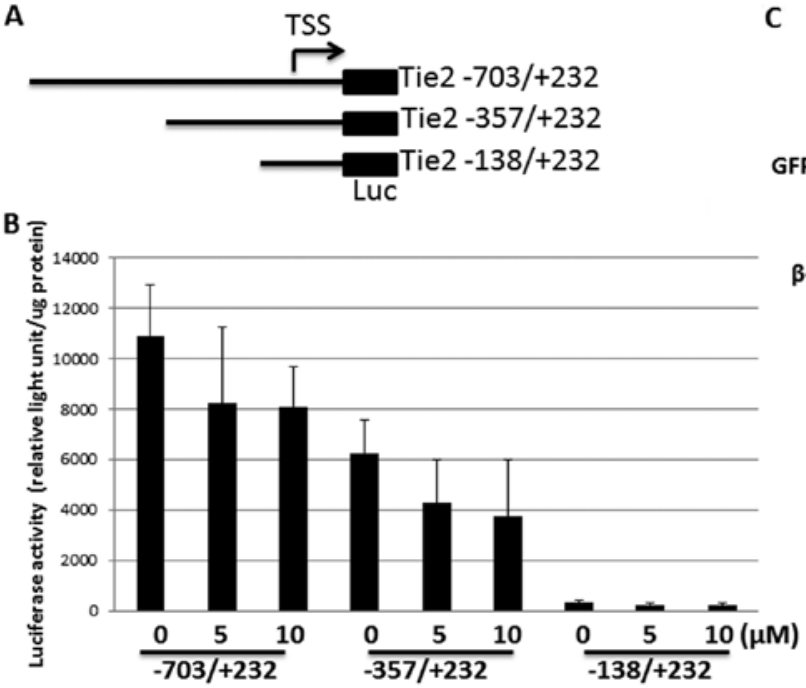

C

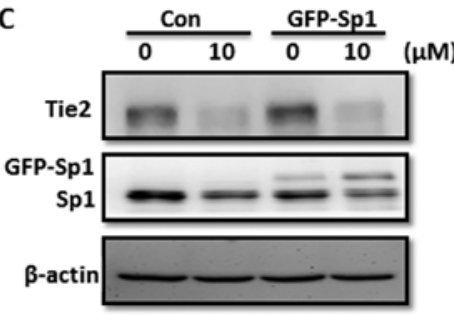

D

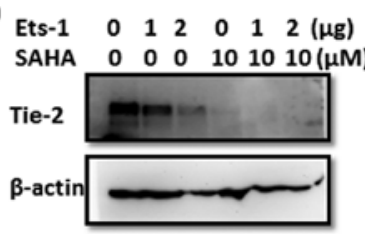

Figure 4. Inhibition of Tie2 promoter activity by SAHA. (A) A scheme showing the constructs of Tie2 promoter-luciferase reporters. TSS, transcriptional start site; Luc, luciferase. (B) FP01 cells were transfected with different reporters and then treated with 5 or $10 \mu \mathrm{M}$ of SAHA for $24 \mathrm{~h}$. Promoter activity was determined and normalized to the cellular protein concentration. (C) Cells were transfected with GFP-tagged Sp1 expression for $48 \mathrm{~h}$ and then treated without or with $10 \mu \mathrm{M}$ of SAHA for another $24 \mathrm{~h}$. Tie2, GFP-Sp1 and Sp1 protein levels were investigated by western blotting. (D) FP01 cells were transfected with 1 or $2 \mu \mathrm{g}$ of Ets-1 for $48 \mathrm{~h}$ and then treated without or with $10 \mu \mathrm{M}$ of SAHA for another $24 \mathrm{~h}$. The Tie2 protein level was studied.

protein was found in the control group suggesting that SAHA increased Tie2 degradation. Treatment of MG132 (proteasome inhibitor) but not chloroquine (autophagy inhibitor) or leupeptin (protease inhibitor) rescued SAHA-induced downregulation of Tie 2 protein. These data suggest the involvement of the ubiquitin/proteasome pathway in Tie 2 degradation (Fig. 5C). A previous study demonstrated that the E3 ligase responsible for the ubiquitination of Tie2 protein is c-Cbl (26). We found that SAHA increased the mRNA level of c-Cbl in a dose-dependent manner (Fig. 6A). The induction was rapidly detected at $6 \mathrm{~h}$ after SAHA treatment and high expression of c-Cbl was persistently found at $24 \mathrm{~h}$ (Fig. 6A). c-Cbl protein was also upregulated dose-dependently in FP01 cells (Fig. 6B). More importantly, knockdown of c-Cbl increased the basal
Tie2 protein level and effectively reversed SAHA-induced downregulation of Tie2 (Fig. 6C). Collectively, we demonstrated that SAHA upregulated c-Cbl expression and induced Tie protein degradation via the ubiquitin/proteasome pathway.

\section{Discussion}

Solid tumors including breast, lung, gastric, head and neck and prostate carcinomas are dependent on angiogenesis and lymphangiogenesis to promote tumor growth and to increase cancer metastasis. In the present study, we provide evidence that the clinically used HDAC inhibitor SAHA suppresses proliferation, sprouting and tube formation of LECs. The anti-angiogenic activity of SAHA was firstly demonstrated by 


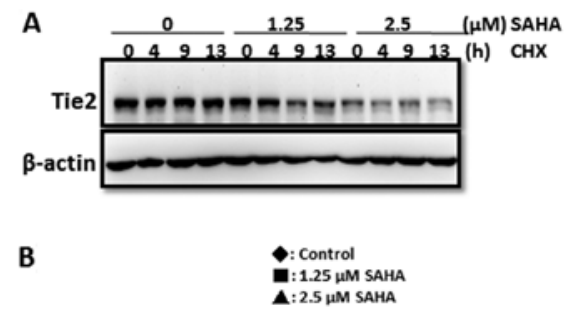

$\begin{array}{lllllll}C & 0 & 1.25 & 2.5 & 0 & 1.25 & 2.5(\mu \mathrm{M}) \mathrm{SAHA}\end{array}$

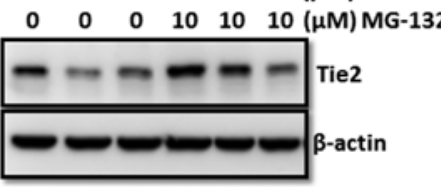

$\begin{array}{llllll}0 & 1.25 & 2.5 & 0 & 1.25 & 2.5(\mu \mathrm{M}) \text { SAHA }\end{array}$

$\begin{array}{llllllll}0 & 0 & 0 & 10 & 10 & 10 & (\mu \mathrm{M}) \mathrm{CQ}\end{array}$
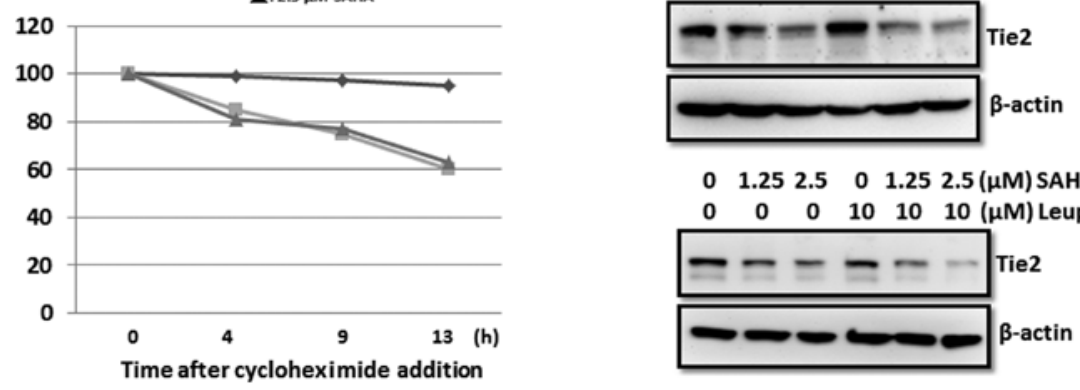

$\begin{array}{llllll}0 & 1.25 & 2.5 & 0 & 1.25 & 2.5(\mu \mathrm{M}) \text { SAHA }\end{array}$

$\begin{array}{lllllll}0 & 0 & 0 & 10 & 10 & 10 & (\mu \mathrm{M}) \text { Leupeptin }\end{array}$

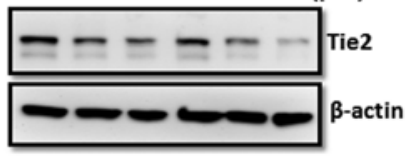

Figure 5. SAHA induces Tie2 protein degradation via the ubiquitin/proteasome pathway. (A) Cells were cultured in media containing various concentrations of SAHA for $24 \mathrm{~h}$ and then treated with $10 \mu \mathrm{g} / \mathrm{ml}$ of cycloheximide (CHX) to block protein synthesis. Cellular proteins were harvested at different times after cycloheximide addition, and the Tie2 protein level was detected by western blotting. (B) The Tie2 protein level in the cells collected at 4,9 and $13 \mathrm{~h}$ was compared to the Tie2 level at time zero (defined as 100\%). (C) Cells were co-treated with different concentrations of SAHA and MG-132 (proteasome inhibitor), chloroquine (autophagy inhibitor) or leupeptin (protease inhibitor). The Tie2 protein level was investigated by western blotting.

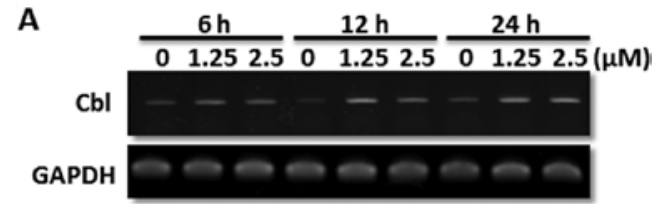

B

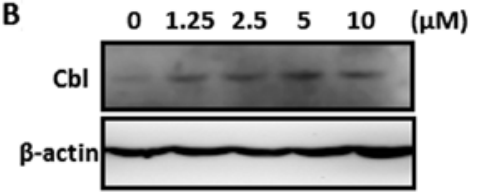

C

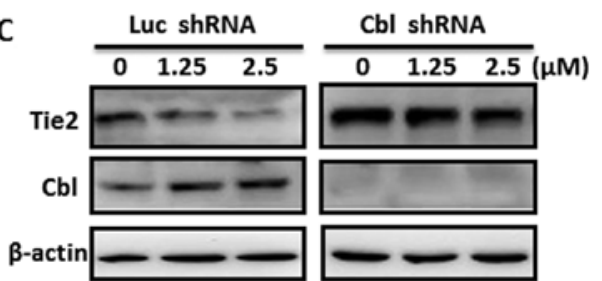

Figure 6. Cbl is involved in SAHA-induced Tie2 degradation. (A) FP01 cells were treated with 1.25 or $2.5 \mu \mathrm{M}$ SAHA for different times, and the $\mathrm{Cbl}$ mRNA level was studied by RT-PCR. (B) The Cbl protein level of FP01 cells treated with different concentrations of SAHA was studied by western blot analysis. (C) FP01 cells were transfected with luciferase (Luc) or Cbl shRNA for $48 \mathrm{~h}$ and then incubated with 1.25 or $2.5 \mu \mathrm{M}$ of SAHA for $24 \mathrm{~h}$. The Tie2 and $\mathrm{Cbl}$ protein levels were investigated.

Deroanne et al (27). By using human umbilical cord endothelial cells (HUVEC) as a model, they showed that Trichostatin-A (TSA) and SAHA prevented VEGF-stimulated HUVECs from invading a type I collagen gel and forming capillary-like structures. In addition, they also found that TSA inhibited the expression of VEGFR1, VEGFR2 and neuropilin-1 induced by VEGF. The in vivo effect of SAHA on angiogenesis was shown by Ugur et al (28). The authors demonstrated that SAHA repressed tumor growth of glioma in an orthotopic animal model. They found that a $30 \%$ reduction in the angiogenesis rate was detected in SAHA-treated animals.
Subsequently, Mühlethaler-Mottet et al (29) also showed that SAHA and TSA strongly impaired hypoxia-induced VEGF expression and secretion in neuroblastoma cells. In addition to the inhibition of pro-angiogenic factors, HDAC inhibitors also stimulated the expression of anti-angiogenic factors to attenuate tumor angiogenesis. For example, a disintegrin and metalloproteinase with thrombospondin motifs 1 (ADAMTS1), an anti-angiogenic proteinase, was upregulated by HDAC inhibitors in lung cancer cells (30). These results support the anti-angiogenic activity of HDAC inhibitors. However, whether HDAC inhibitors can inhibit lymphangiogenesis is unclear. Induction of tumor lymphangiogenesis consists of two main steps. First, cancer cells secrete pro-lymphangiogenic factors including VEGF-A, VEGF-C and fibroblast growth factor-2 to stimulate the proliferation of quiescent LECs. Second, the stimulated LECs sprout from the lymphatic vessels, migrate toward tumors and form a new tube structure. Thus, both cancer cells and LECs are important for lymphangiogenesis. We recently demonstrated that SAHA attenuated the expression and production of VEGF-C in breast cancer cells (31). We now demonstrate that SAHA also suppresses the proliferation, sprouting and tube formation of LECs. Our results suggest that SAHA is a potent anti-lymphangiogenic drug by inhibiting both cancer cells and LECs.

Another important finding of this study is the identification of Tie2 as a molecular target of SAHA. The Ang/Tie signaling pathway is known to play a crucial role in angiogenesis and lymphangiogenesis. Therefore, several strategies have been developed to inhibit this signaling pathway. The first strategy is targeting the Tie 2 receptor. To date, a number of Tie 2 kinase inhibitors have been tested in cell-based assays or in animal studies (32). However, only a few compounds have advanced to clinical trials. For example, CEP-11981 is now undergoing Phase I study in patients with advanced solid tumors. However, this drug inhibits Tie2, VEGFRs and FGFRs. Thus, it is not truly Tie2 selective. The second strategy is Ang1 or Ang2 traps. For example, AMG-386 is a peptibody developed to 
target both Ang1 and Ang2 (33). Peptibodies, an alternative therapeutic format to monoclonal antibodies, consist of biologically active peptides grafted onto an Fc domain. This design has benefits to increase selectivity by the active peptide domain and to retain the desirable features of antibodies to prolong plasma residency time $(34,35)$. AMG-386 is now undergoing Phase I and II trials, either alone or in combination with chemotherapeutic drugs, for the treatment of various cancers (36). In the present study, we explored SAHA as a new Tie 2 inhibitor by downregulating its expression in LECs. In addition, we elucidated the underlying mechanisms and found that SAHA suppressed Tie2 via repression of gene transcription and promotion of protein degradation via the ubiquitination/proteasome pathway.

\section{Acknowledgements}

This study was supported by the grants: DOH 101-TD-C111-002 and DOH 101-TD-C-111-004 from the Department of Health, Taiwan, to W.-C. H.

\section{References}

1. Minucci S and Pelicci PG: Histone deacetylase inhibitors and the promise of epigenetic (and more) treatments for cancer. Nat Rev Cancer 6: 38-51, 2006.

2. Inche AG and La Thangue NB: Chromatin control and cancerdrug discovery: realizing the promise. Drug Discov Today 11: 97-109, 2006.

3. Olsen EA, Kim YH, Kuzel TM, et al: Phase IIb multicenter trial of vorinostat in patients with persistent, progressive, or treatment refractory cutaneous T-cell lymphoma. J Clin Oncol 25: 3109-3115, 2007

4. Duvic M and Vu J: Vorinostat: a new oral histone deacetylase inhibitor approved for cutaneous T-cell lymphoma. Expert Opin Investig Drugs 16: 1111-1120, 2007.

5. Emanuele S, Lauricella M and Tesoriere G: Histone deacetylase inhibitors: apoptotic effects and clinical implications (Review) Int J Oncol 33: 637-646, 2008

6. Zhao Y, Tan J, Zhuang L, et al: Inhibitors of histone deacetylases target the Rb-E2F1 pathway for apoptosis induction through activation of proapoptotic protein Bim. Proc Natl Acad Sci USA 102: 16090-16095, 2005.

7. Gui CY, Ngo L, Xu WS, et al: Histone deacetylase (HDAC) inhibitor activation of $\mathrm{p} 21^{\mathrm{WAF} 1}$ involves changes in promoterassociated proteins, including HDAC1. Proc Natl Acad Sci USA 101: 1241-1246, 2004.

8. Richon VM, Sandhoff TW, Rifkind RA and Marks PA: Histone deacetylase inhibitor selectively induces $\mathrm{p} 21^{\mathrm{WAF} 1}$ expression and gene-associated histone acetylation. Proc Natl Acad Sci USA 97: 10014-10019, 2000.

9. Woan KV, Sahakian E, Sotomayor EM, Seto E and Villagra A: Modulation of antigen-presenting cells by HDAC inhibitors: implications in autoimmunity and cancer. Immunol Cell Biol 90 55-65, 2012.

10. Blanchard F and Chipoy C: Histone deacetylase inhibitors: new drugs for the treatment of inflammatory diseases? Drug Discov Today 10: 197-204, 2005.

11. Deroanne CF, Bonjean K, Servotte S, et al: Histone deacetylases inhibitors as anti-angiogenic agents altering vascular endothelial growth factor signaling. Oncogene 21: 427-436, 2002.

12. Alitalo K: The lymphatic vasculature in disease. Nat Med 17: 1371-1380, 2011.

13. Achen MG, McColl BK and Stacker SA: Focus on lymphangiogenesis in tumor metastasis. Cancer Cell 7: 121-127, 2005.

14. Cao R, Björndahl MA, Religa P, et al: PDGF-BB induces intratumoral lymphangiogenesis and promotes lymphatic metastasis. Cancer Cell 6: 333-345, 2004.

15. Augustin HG, Koh GY, Thurston G and Alitalo K: Control of vascular morphogenesis and homeostasis through the angiopoietin-Tie system. Nat Rev Mol Cell Biol 10: 165-177, 2009.
16. Maisonpierre PC, Suri C, Jones PF, et al: Angiopoietin-2, a natural antagonist for Tie2 that disrupts in vivo angiogenesis. Science 277: 55-60, 1997.

17. Yuan HT, Khankin EV, Karumanchi SA and Parikh SM: Angiopoietin 2 is a partial agonist/antagonist of Tie2 signaling in the endothelium. Mol Cell Biol 29: 2011-2022, 2009.

18. Kim I, Kim JH, Moon SO, Kwak HJ, Kim NG and Koh GY: Angiopoietin-2 at high concentration can enhance endothelial cell survival through the phosphatidylinositol 3'-kinase/Akt signal transduction pathway. Oncogene 19: 4549-4552, 2000.

19. Murray BW,Padrique ES, Pinko C and McTigue MA: Mechanistic effects of autophosphorylation on receptor tyrosine kinase catalysis: enzymatic characterization of Tie2 and phospho-Tie2. Biochemistry 40: 10243-10253, 2001.

20. Jones N, Chen SH, Sturk C, Master Z, Tran J, Kerbel RS and Dumont DJ: A unique autophosphorylation site on Tie2/Tek mediates Dok-R phosphotyrosine binding domain binding and function. Mol Cell Biol 23: 2658-2668, 2003.

21. Jones N, Master Z, Jones J, Bouchard D, Gunji Y, Sasaki H, Daly R, Alitalo K and Dumont DJ: Identification of Tek/Tie2 binding partners. Binding to a multifunctional docking site mediates cell survival and migration. J Biol Chem 274: 30896-30905, 1999.

22. Dellinger M, Hunter R, Bernas M, Gale N, Yancopoulos G, Erickson $\mathrm{R}$ and Witte M: Defective remodeling and maturation of the lymphatic vasculature in Angiopoietin-2 deficient mice. Dev Biol 319: 309-320, 2008.

23. Shimoda H, Bernas MJ, Witte MH, Gale NW, Yancopoulos GD and Kato S: Abnormal recruitment of periendothelial cells to lymphatic capillaries in digestive organs of angiopoietin-2-deficient mice. Cell Tissue Res 328: 329-337, 2007.

24. Kajiya K, Kidoya H, Sawane M, Matsumoto-Okazaki Y, Yamanishi H, Furuse M and Takakura N: Promotion of lymphatic integrity by angiopoietin-1/Tie2 signaling during inflammation. Am J Pathol 180: 1273-1282, 2012.

25. Pan MR, Chang TM, Chang HC, Su JL, Wang HW and Hung WC: Sumoylation of Prox 1 controls its ability to induce VEGFR3 expression and lymphatic phenotypes in endothelial cells. J Cell Sci 122: 3358-3364, 2009.

26. Wehrle C, Van Slyke P and Dumont DJ: Angiopoietin-1-induced ubiquitylation of Tie 2 by c-Cbl is required for internalization and degradation. Biochem J 423: 375-380, 2009.

27. Deroanne CF, Bonjean K, Servotte S, et al: Histone deacetylases inhibitors as anti-angiogenic agents altering vascular endothelial growth factor signaling. Oncogene 21: 427-436, 2002.

28. Ugur HC, Ramakrishna N, Bello L, et al: Continuous intracranial administration of suberoylanilide hydroxamic acid (SAHA) inhibits tumor growth in an orthotopic glioma model. J Neurooncol 83: 267-275, 2007.

29. Mühlethaler-Mottet A, Meier R, Flahaut M, et al: Complex molecular mechanisms cooperate to mediate histone deacetylase inhibitors anti-tumour activity in neuroblastoma cells. Mol Cancer 7: 55, 2008.

30. Chou CW and Chen CC: HDAC inhibition upregulates the expression of angiostatic ADAMTS1. FEBS Lett 582: 4059-4065, 2008.

31. Cheng HT and Hung WC: Inhibition of lymphangiogenic factor VEGF-C expression and production by the histone deacetylase inhibitor suberoylanilide hydroxamic acid in breast cancer cells. Oncol Rep 29: 1238-1244, 2013.

32. Huang H, Bhat A, Woodnutt $G$ and Lappe R: Targeting the ANGPT-TIE2 pathway in malignancy. Nat Rev Cancer 10: 575-585, 2010.

33. Oliner J, Min H, Leal J, et al: Suppression of angiogenesis and tumor growth by selective inhibition of angiopoietin-2. Cancer Cell 6: 507-516, 2004

34. Shimamoto G, Gegg C, Boone T and Quéva C: Peptibodies: A flexible alternative format to antibodies. MAbs 4: 586-591, 2012.

35. Beck A and Reichert JM: Therapeutic Fc-fusion proteins and peptides as successful alternatives to antibodies. MAbs 3: 415-416, 2011.

36. Herbst RS, Hong D, Chap L, et al: Safety, pharmacokinetics and antitumor activity of AMG 386, a selective angiopoietin inhibitor, in adult patients with advanced solid tumors. J Clin Oncol 27: 3557-3565, 2009. 